SERIAL COMPOSITION OF 2-WAY FINITE-STATE TRANSDTCERS

AND SIMPLE PROGRAMS ON STRINGS

\author{
Nichal P. Chytil \\ Vojtĕch Jákl \\ Charles University \\ Malostranské nám. 25 \\ 11800 Praha 1 - M. Strana \\ Czechoslovakia
}

Introduction

Hopcroft and Ullman [1] described an algorithm for reverse run of deterministic generalized sequential machines. This algorithm does not go beyond abilities of 2-way finite automata. The algorithm was then used [2] in the proof of the fact that serial composition of a deterministic gsm $A_{1}$ and a 2- way deterministic finite-state transducer $\mathrm{A}_{2}$ can be replaced by a single 2-way deterministic finite-state transducer, say $\mathrm{A}_{3}$. Let us recall the main idea of the proof (c.f. fig. I).
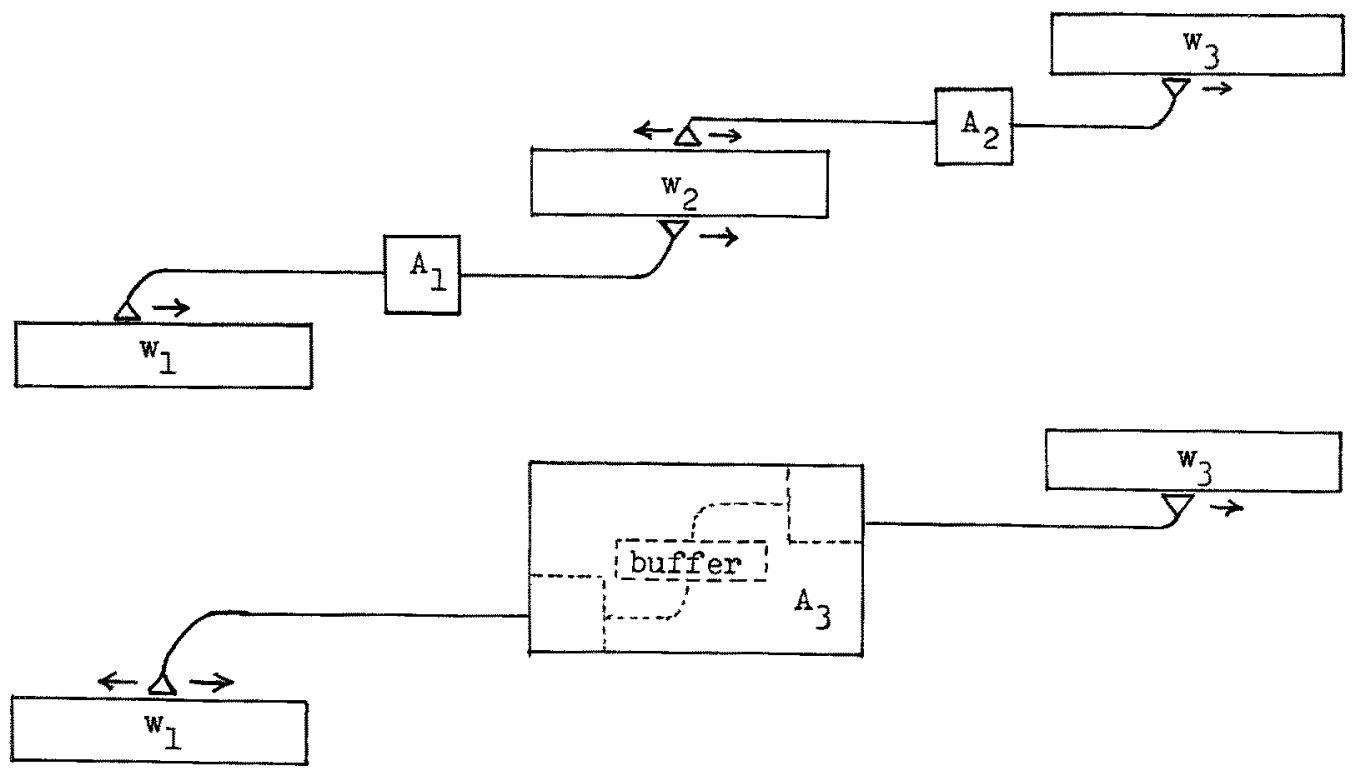
$\mathrm{A}_{3}$ in fact simulates step by step the computation of $\mathrm{A}_{2}$ on the tape $w_{2} \cdot A_{3}$ has not, however, all the tape $w_{2}$ at his disposal. It has only a segment of $w_{2}$ stored in a finite buffer. It is the segment which is in the given moment scanned by $A_{2}$. The input head of $A_{3}$ is placed at the square of $\mathrm{w}_{1}$ which was translated by $\mathrm{A}_{1}$ into the segment of $\mathrm{w}_{2}$ currently stored in the buffer. If $\mathrm{A}_{2}$ moves its head to the right, $\mathrm{A}_{3}$ updates the content of the buffer moving its head rightwards and simulating one or more following steps of $A_{1}$. If $A_{2}$ moves its head leftwards, then $A_{3}$ updates the content of the buffer employing the algorithm for reverse run of $\mathrm{A}_{1}$ and reconstructs one or more preceding steps of $A_{1}$.

In this paper we introduce an algorithm for reverse run of more general kind of machines, so called quasideterministic gsms. This algorithm is then employed in the proofs of some results about 2-way finite-state transducers and the functions computed by them.

The fact that the functions computable by 2-way finite transducers an closed under composition is then used in investigation of simple programs on strings.

\section{Quasideterministic devices}

A nondeterministic gsm $A$ is quasideterministic if for every input w there is at most one computation over $w$ beginning in an initial state and reaching a final state.

Then for every mapping (as defined e.g. in (3]) $A: X^{*} \rightarrow P\left(Y^{*}\right)$ performed by a quasideterministic $\operatorname{gsm} A$ is $\operatorname{card} A(w) \leq 1$ for every $\mathrm{w} \in \mathrm{X}^{*}$. Such a mapping can be in an obvious way identified with a (partial) mapping $A: X^{*} \rightarrow Y^{*}$. Every mapping of this kind will be called quasideterministic gsm-mapping in this paper.

Example 1 . Let $g$ be a $1-1$ mapping computable by a sequential machine. Then the inverse mapping $g^{-1}$ is a quasideterministic gsm mapping.

Outline of the proof. In the state diagram of the Mealy sequential machine M performing $g$ "reverse arrows" and interchange the role of input and output symbols. The results is a quasideterministic gsm for $\mathrm{g}^{-1}$.

Example 2.

This example is connected with deterministic 2-way finite-state transducers. 
By a deterministic 2-way finite-state transducer (2-DFT) we shall mean a deterministic device with finite-state control unit, 2-way read - only input head and a l-way output head. The output head can print a word in an output alphabet in every step of computation. We suppose that the input words are bounded by a pair of endmarkers $\not \notin, \not$. Every computation starts in the initial state on the left endmarker. It stops by leaving the input tape. Every 2-DFT determines a (partial) function computable by 2-DFT.

By the history for 2-DFr II and an input word $\not x_{1} \ldots x_{n} \not$ we mean a word

$\operatorname{Hist}(\mathrm{H}, \mathrm{w})=$ $=\left(\not 2, q_{01}, q_{02}, \ldots, q_{0 r_{0}}\right)\left(x_{1}, q_{11}, \ldots, q_{1 r_{1}}\right) \ldots\left(\not, q_{n+1}, 1, \ldots, q_{n+1, r_{n+1}}\right)$, where $q_{i j} \in Q \times\{L, R\} . q_{i j}=(q, I)$ (or $q_{i j}=(q, R)$ ) means that visiting the $i-t h$ square for the $j$-th time, $M$ is in state $q$ and enters the square from the left (or from the right).

If the computation of $\mathbb{M}$ on $w$ is nonterminating, then Hist $(\mathbb{N}, w)$ is undefined.

Lemma 1. The mapping

$\mathrm{w} \longrightarrow \mathrm{Hist}(\mathrm{M}, \mathrm{w})$

is a quasideterministic mapping for every 2-DFI .

Outline of the proof. By records we shall mean the elements composing histories, i.e. records are sequences of the form $\left(x, q_{1}, \ldots, q_{2}\right)$, where $x \in X \cup\{\not, \mathscr{f}\}$ and $q \in Q \times\{I, R\}$. For arbitrary $\mathbb{N}$ there is only a finite number of records which can occur in some terminating computation, because no square can be visited two times in the same state. The set $R$ of these admissible records is therefore finite.

Let us construct a gsm $G$ whose state-space and output alphabet are both equal to $R$. The control function $\delta$ of $G$ is defined as follows:

$$
\left[\left(x, q_{1}, \ldots, q_{s}^{\prime}\right),\left(x, q_{1}, \ldots, q_{r}\right)\right] \in \delta\left(\left(x, q_{1}, \ldots, q_{r}\right), z\right) \Leftrightarrow \text { df }
$$

$\Leftrightarrow \quad x=z \&\left[\left(x^{\prime}, q_{1}^{\prime}, \ldots, q_{s}^{\prime}\right)\right.$ can occur as the right neighbour of $\left(x, q_{1}, \ldots, q_{r}\right)$ in some history of a computation]

In every pair $\left(r_{1}, r_{2}\right) \in J(\ldots), r_{1}$ represents the next state and $r_{2}$ the symbol to be printed.

The set $S \subseteq R$ of the initial states of $G$ is formed by all records with the first component equal to $\not$ and the final set 
$F \subseteq R$ of $G$ is formed by all records with the first component equal to $\not$.

Every successful computation of $G$ for input $w$ outputs a history of a successful computation of M over $w$. Since $M$ is deterministic, there is at most one such a computation. Different computations of G yield. different outputs and therefore there is at most one successful computation for any input $w$. $G$ is quasideterministic.

A nondeterministic gsm $G_{1}$ is dual to a nondeterministic gsm $G_{2}$ iff the state diagram of $G_{1}$ can be obtained from the state diagram of $G_{2}$ by reversing arrows and interchanging the role of initial and final states.

The following lemma is obvious.

Lemma 2. The nondeterministic gsm dual to a quasideterministic gsm is quasideterministic.

Let us describe the algorithm for reverse run of quasideterministic gsm.

Let $G_{I}$ be a quasideterministic gsm, $Q, X, Y, \delta, S$ and $F$ its statespace, input alphabet, output alphabet, control function, initial and final set, respectively. Let $G_{2}$ be the gsm dual to $G_{1}$. $G_{2}$ is quasideterministic by Iemma 2. Let $D_{1}$ be the deterministic finite acceptor derived from $G_{1}$ by the standard subset construction, $D_{2}$ the deterministic acceptor derived by the subset construction from $G_{2}$.

Then the state of $D_{1}$ after reading $\not x_{1} \ldots x_{i-1}$ is equal to the set $K_{I}^{i}=\delta\left(s, \not \subset x_{1} \ldots x_{i-1}\right)=\{q \in Q ; q$ is accessible by $Q$ from an initial The state of $D_{2}$ after scanning $\not x_{n} x_{n-1} \cdots x_{1}$ is equal to the set $K_{2}^{i}=\left\{q \in Q ; G_{2}\right.$ can access $q$ from a state of $F$ by reading $\left.\delta x_{n} \cdots x_{i}\right\}$ Since $G_{1}$ is quasideterministic, card $\left(K_{1}^{i} \cap K_{2}^{i}\right) \leq 1$. The set $K_{1}^{i} \cap K_{2}^{i}$ contains one element iff there exists a successful computation of $G_{1}$ for input $\not x_{1} \ldots x_{n} \not s$ (cf. Fig. 2).

Now it is easy to see how the algorithm for the reverse run of $G_{1}$ works. It keeps $\mathrm{K}_{1}^{i}$ and $\mathrm{K}_{2}^{i}$ in its finite memory, when the head is visiting the $i$-th square. $\mathrm{K}_{1}^{i} \cap \mathrm{K}_{2}^{i}$ determines uniquely the state of $G_{1}$ during the successful computation, if there is any.

To reconstruct the situation of $G_{1}$ at the $i-1$-th square, it is 


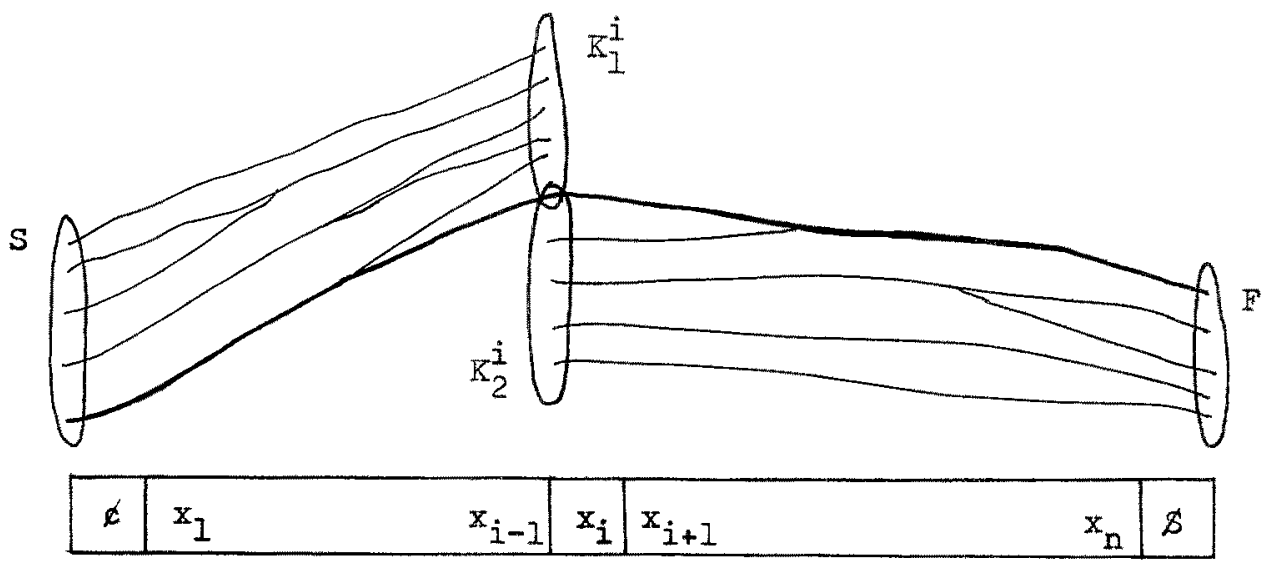

Fig. 2

sufficient to simulate one step of the deterministic automaton $D_{2}-$ $-\mathrm{K}_{2}^{\mathrm{i}-1}$ is constructed - and to reconstruct $\mathrm{K}_{1}^{i-1}$. Since $\mathrm{D}_{1}$ is deterministic, $K_{1}^{i-1}$ can be reconstructed employing the algorithm of Hopcroft and Ullman mentioned in the introduction to this paper.

All this apparently can be done by a 2-DFT. Evaluation of the next state of $G_{1}$ is the same problem as computing one step in the reverse run of $G_{2}$ and is solved by the dual procedure.

The algorithm starts by computing $\mathrm{K}_{1}^{1}$ and $\mathrm{K}_{2}^{1}$. It makes no problem, as $K_{1}^{1}=\delta(S, \not)$ is a fixed set and $K_{2}^{1}$ is computed by scanning the tape backwards and simulating $\mathrm{D}_{2}$.

Theorem 1. If $g$ is a quasideterministic gsm mapping and if $f$ is $a$ mapping computable by a 2-DFT, then $f \cdot g$ is computable by a 2-DFT.

The proof of the theorem is quite analogous to the proof of the theorem by Aho, Hopcroft and Jlinan [2] outlined in the introduction. It suffices to replace the algorithm for reverse run of deterministic gsm by the above described algorithm.

Recall a lemma from [4].

Lemma 3 (Aho, UIIman). Let $H_{1}$ be a 2-DFT computing a function $f$. Let $g$ be another function computable by 2-DFT. Then there is a 2-DFT $\mathrm{M}_{2}$ computing a function $h$ such that

$$
h\left(H i s t\left(M_{1}, w\right)\right)=g \circ f(w), \quad \text { for all } w \text {. }
$$


Lemma 1, Theorem 1 and Lemma 3 immediately imply the following theorem.

Theorem 2. (Aho, Jllman [4]) The class of functions computable by 2-DFT is closed under composition of functions.

We close the paragraph by two results which can be proved analogousIy to Theorem 2 using appropriate modifications of Lemma 1.

Corollary. Let $f$ be a function computable by a deterministic boundedcrossing transducer (cf. (5)) and let $g$ be a function computable by 2-DFT. Then $g \circ f$ is computable by 2-DFT.

By quasideterministic 2-way finite transducer (2-QFW) we shall mean a nondeterministic 2-way finite transducer with the property that for every input word chw there is at most one terminating computation starting on $\not \subset$ in an initial state.

Coroliary. Let $f$ be computable by $2-Q \mathrm{QPT}$ and let $g$ be computable by 2-DFT. Then $g \cdot f$ is computable by 2-DFT.

Consequently, a function is computable by $2-Q F T$ iff it is computable by 2-DFT.

Simple programs on strings

Various kinds of automata. have been investigated as devices for recognizing and generating languages. On the other hand, D. Scott (6) expressed the idea that "functions are better than sets", i.e. that investigation of functions computable by automata could prove to be in some aspects more fruitful approach. The results of this paragraph support the idea.

We shall define a simple programming language IOOP 2 which is a little more powerful than the language LOOP and the language LOOP with weak sum introduced and studied by Ausiello (7) and Ausiello Moscarini [8]. We shall prove that the functions programmable in IOOP 2 are exactly the functions computable by 2-DFT. The existence of such a language bears upon the fact that the class of functions is closed under composition. For, if $P_{1} ; P_{2}$ should be a program whenever $P_{1}$ and $P_{2}$ are programs, then the closure under composition of functions is ustually unavoidable. 
To express the semantics of LOOP 2 - programs, we have to consider each identifier in a program to be the name of a register containing a word in a given alphabet. We assume, besides, the existence of a special control register, which is used during execution of loops. Single symbols of strings in the control register are accessible for inspecting (but not rewriting) through a "window" which can be moved backwards and forwards.

Then the LOOP 2 programs operating on strings in an alphabet $X=$ $=\left\{x_{1}, \ldots, x_{n}\right\}$ can be defined inductively as follows. We explain the semantics paralelly with the syntax of the programs.

1) elementary statements

$\begin{array}{ll}\text { a) }\langle i d\rangle:=\varepsilon & \text { : clear register }\langle i d\rangle \\ \text { b) }\left\langle i d_{1}\right\rangle:=\left\langle i d_{2}\right\rangle & \text { : store the content of }\left\langle i d_{2}\right\rangle \text { in }\left\langle i d_{1}\right\rangle \\ \text { c) }\langle i d\rangle:=\langle i d\rangle_{0} "\langle\text { const }\rangle ": \text { compute the right } \text { : const -successor }\end{array}$ of the word contained in 〈id〉

(i.e. the word consisting of the word

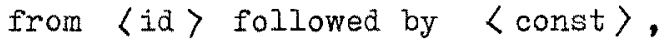
where 〈const $\rangle$ is a word in $X)$ and store the result in 〈id〉.

(We shall refer to statements of the types a) - c) as to basic statements.)

d) Left

e) right

f) (conditional statements)

if $\langle i d\rangle \neq \varepsilon$ then $s_{1} ; \ldots ; s_{n}$ else $s_{n+1} ; \ldots ; s_{m} \frac{f i}{\text { are }}$

where $s_{1}, \ldots s_{m}$ are elementary statements

if $\langle i d\rangle \neq \varepsilon$ then $S_{I} ; \ldots ; S_{m}$ fi
: move the window of the control register one position leftwards

: move the window one position rightwards

: if the register $\langle i d\rangle$ is nonempty then the sequence of statements $s_{1}, \ldots, s_{n}$ is executed, $S_{n+1} ; \ldots ; S_{m}$ are executed otherwise.

2) elementary programs

An elementary program is every program of the form $s_{1} ; \ldots s_{n}$, where $\mathrm{s}_{1}, \ldots, \mathrm{s}_{\mathrm{n}}$ are elementary statements.

3) LOOP 2 programs

LOOP 2 programs willbe also called simple programs in the sequel. A simple progranis each program satisfying one of the following conditions. 
i) $P$ is an elementary program,

ii) $P$ is of the form

$$
\begin{gathered}
\text { LOOP }\langle i d\rangle \\
x_{1}: P_{1} \\
\ldots \\
x_{n}: P_{n} \\
\text { END, }
\end{gathered}
$$

where $\mathrm{P}_{1}, \ldots, \mathrm{P}_{\mathrm{n}}$ are elementary programs,

iii) $P$ is of the form $P_{1} ; P_{2}$, where $P_{1}, P_{2}$ are simple programs.

The program LOOP 〈id〉

$$
\begin{gathered}
\mathrm{x}_{1}: \mathrm{P}_{1} \\
\ldots \\
\mathrm{x}_{n}: \mathrm{P}_{\mathrm{n}}
\end{gathered}
$$

END

is interpreted in the following way:

- the content of 〈id〉 (a string $w$ ) is stored in the control register and the control window is moved to the first symbol of the string $w$;

- while the window is attached to some symbol of $w$ execute

all the program $P_{i}$, if the window displays the symbol $x_{i}$ (i.e. the statements right and left within $P_{i}$ do not influence the running execution of $P_{i}$ ).

Theorem 3. All functions computable by 2-DFW can be programmed in IOOP 2.

Proof. Let $\mathrm{N}$ be a $2-\mathrm{DFT}$ and $Q=\left\{q_{0}, \ldots, \mathrm{q}_{\mathrm{m}}\right\}, \mathrm{X}_{1}, \mathrm{X}_{2}$, $\delta: Q \times X_{1} \longrightarrow Q \times X_{2}^{*} \times$ \{left, right\} and $q_{0}$ its state space, input alphabet, output alphabet, control function and the initial state, respectively. Denote $\mathrm{x}=\mathrm{x}_{1} \cup \mathrm{x}_{2}=\left\{\mathrm{x}_{1}, \ldots, \mathrm{x}_{n}\right\}$. Then the following simple program evidently computes the same function as M.

IN U; OUT V; \{declaration of input and output register - U contains the input string at the start of the computations, the other registers are empty. $V$ contains the output word at the end of the computation

$Q_{0}:=x_{1}$

LOOP U $\quad$ simulation of $\mathrm{M}\}$

$x_{1}: P_{1}$

$x_{n}: P_{n}$

END

lend of the program

where 
for $x_{i} \notin X_{1}$ is $P_{i}=$ left; right infinite cycle\}

for $x_{i} \in X_{1}$ is $P_{i}=S_{i 0} ; \ldots ; S_{i m}$ and

$S_{i j}=$ if $Q_{j} \neq \varepsilon$ then $Q_{j}:=\varepsilon ; Q_{K}:=x_{I} ; V:=V_{*}$ "v"; $S \underline{f i}$, where

$\mathrm{S} \in\{\underline{\text { right}}, \underline{\text { left }}\}$ and $\mathrm{k}, \mathrm{v}$ and $\mathrm{S}$ are such that

$\delta\left(q_{j}, x_{i}\right)=\left(q_{k}, v, s\right)$.

Theorem 4. All functions programmable in LOOP 2 are computable by 2-DFT.

Proof. Let $P$ be a simple program and $X_{I}, \ldots, X_{m}$ all identifiers occurring in $P$. Suppose that all the identifiers are declared as input and at the same time as output ones. Then $P$ defines a partial transformation of $\left(X^{*}\right)^{\mathrm{m}}$.

We shall construct a 2-DFT $A_{p}$ performing the same transformation as $P$ in the following sense: input and output tapes of $A_{P}$ have $m$ tracks. Input m-tuple of strings $\left(w_{1}, \ldots, w_{m}\right)$ is encoded on the input tape as indicated in Fig. 3 .

\begin{tabular}{|c|c|c|c|}
\hline$w_{1}$ & \multicolumn{3}{|c|}{ empty } \\
\hline empty & $w_{2}$ & \multicolumn{2}{|c|}{ empty } \\
\hline empty & $w_{3}$ & empty \\
\hline$\ldots$. & & $w_{m}$ \\
\hline
\end{tabular}

Fig. 3

The output m-tuple is encoded in the same way.

Let us describe the construction of $A_{P}$ inductively, following the inductive definition of simple programs.

1) $P$ is elementary

We shall describe three 2-DFr $A_{1}, A_{2}, A_{3}$ such that the serial composition of $A_{1}, A_{2}$ and $A_{3}$ will perform the same transformation as $P$.

Automaton $A_{1}: A_{1}$ has the program $P$ stored in its finite control unit. 
It also disposes of an auxiliary m-bit memory $E$. In every moment, the $i-t h$ bit of $E$ is set to $I$ iff the register $X_{i}$ of $P$ is nonempty in a corresponding moment of the execution of $P$.

At the beginning of the computation, $A_{1}$ inspects the input tape and learns which tracks are completely empty. This information is stored in $\mathrm{E}$.

Then it prints out the input tape. After it (i.e. on the right of the string $w_{m}$ in the m-th track), it prints a sequence of basic statements $s_{1}, \ldots, S_{r}$. This sequence describes step by step all actions of $P$ changing the content of registers. A constructs the sequence as follows:

it goes step by step through the program $P$ stored in its finite memory and

- when it reaches $a$ basic statement $S$ in $P$, it prints $S$. Basic statements can clear a nonempty register or vice versa. Therefore $A_{1}$ updates $E$ when printing $S$;

- it omits statements right and left (because they have no meaning in elementary programs);

- when a conditional statement is to be executed in $P, A_{1}$ decides (by inspecting $E$ ) whether the test condition of the statement holds or not and enters the corresponding branch of the program $P$. The final form of the output tape is illustrated by Fig. 4 .

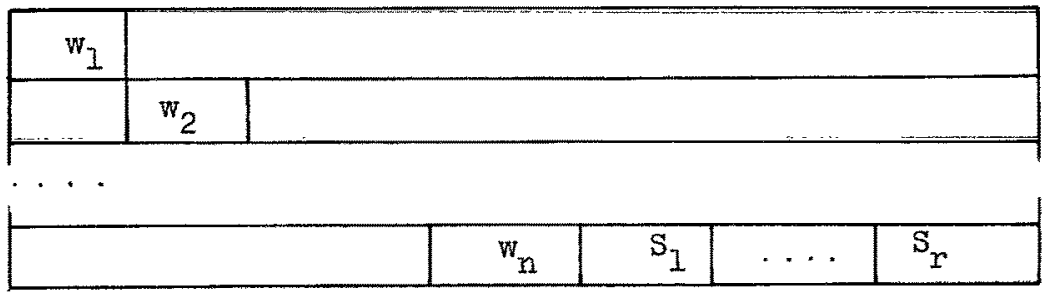

Fig. 4

Automaton $\mathrm{A}_{2}$ : $\mathrm{A}_{2}$ disposes of an auxiliary finite memory $\mathrm{R}$ divided into $m$ squares. Each of the squares can contain one of the symbols $1,2, \ldots$ $\ldots, \mathrm{m}$,erase. The content of $\mathrm{R}$ will be interpreted as references of registers to other registers. Denote the content of the $i-t h$ square by $x_{i}$. Then e.g. the reference $x_{i}=j$ means that $x_{i}$ should be treated as $x_{j}$.

At the beginning of the computation, $A_{2}$ sets $x_{i}=i$ for all $i$. Then $A_{2}$ is scanning the input tape leftwrds and executing the statements $\mathrm{S}_{r}, \ldots, \mathrm{S}_{1}$ (in this order). Execution or more precisely interpreting of the statements of the type $X_{j}:=\varepsilon$ and $X_{j}:=X_{k}$ consists in changing 
the content of the memory $R$ while the remaining type of basic statements, i.e. $\mathrm{X}_{i}:=\mathrm{X}_{1} 0^{\prime}$ 〈const>" causes an output printing. More precisely:

- scanning $X_{i}:=\varepsilon, A_{2}$ assigns erase to each $x_{k}$ such that $x_{k}=i$ and makes a left shift,

- scanning $\mathrm{X}_{i}:=\mathrm{x}_{j}, A_{2}$ executes assignements $\mathrm{x}_{k}:=j$, for all $\mathrm{x}_{k}$ equal to $i$ and makes a left shift,

- scanning $X_{i}:=X_{i} \cdot " u$ ", $A_{2}$ prints $u$ in the $k$-th track, for all $k$ such that $x_{k}=i$ and also proceeds leftwards.

When $A_{2}$ reaches the segment of the tape on the left of $S_{1}$, then it continues going leftwards and in each step the symbol which is in the $k$-th track on the input tape is printed into the i-th output track whenever $\mathrm{x}_{i}=\mathrm{k}$; blank is printed into the $i$-th track for $\mathrm{x}_{i}=$ erase.

Let us summarize the main idea of this procedure. If the program $P$ assigns $X_{i}:=X_{j}$ in the $s-t h$ step of computation, then all the history of $x_{i}$ up to the $s-t h$ step is in fact catelled and replaced by the history of $x_{j}$ up to the $s-t h$ step. Similarly, the statement $X:=\varepsilon$ means that $x_{i}$ is cleared irrespective of all its history up to the s-th step.

Therefore, the automaton $A_{2}$ traverses the history of the execution of $P$ backwards and if it has $x_{i}=j$ in its table of references $R$ when scanning $S_{S}$ then for its following computation in the $i-t h$ track, the computation in the $j-t h$ track is authoritative. If $x_{i}=$ erase, then the remaining segment of the $i-t h$ track is to be erased.

When $A_{2}$ finishes its work, its output tape contains the final content of $x_{i}$ in the $i$-th output track $(1 \leq i \leq m)$. The output, however, need not be in the desired normalized form and blank segments can be scattered between symbols in each track.

Automaton $\mathrm{A}_{3}: \mathrm{A}_{3}$ rearranges the tape in the normalized fashion, i.e. it prints out the content of the first input track into the first output track omitting the blanks, then it prints the content of the second. track and so on. (Recall Fig. 3.)

The serial composition of $A_{1}, A_{2}, A_{3}$ performs the same transformation of $\left(X^{*}\right)^{m}$ as $P$. By Theorem 2, the serial composition of $A_{1}, A_{2}$ and $A_{3}$ can be replaced by a single 2-DFT $A_{P}$.

2) $P$ is of the form LOOP $x_{1}$

$$
\begin{gathered}
x_{1}: P_{1} \\
\ldots \\
x_{n}: P_{n}
\end{gathered}
$$


The equivalent 2-DFT $A_{P}$ can be again described by a serial compositition of three 2-DFTs $A_{1}^{\prime}, A_{2}, A_{3} \cdot A_{2}$ and $A_{3}$ are the same as in the preceding case. $A_{1}^{\prime}$ is a modification of $A_{1}$. It starts by initializing $E$ and by reprinting the input tape in the same way as $A_{1}$. Then $A_{I}$ moves its head to the first symbol of $w_{1}$. If the first symbol is $x_{1}$, it starts by executing $P_{i}$ similarly as $A_{1}$ executes all $P$ with the exception that the statements right and left are not neglected as in the previous case. They are inerpreted as instructions controlling the moves of the head. If the way through $P_{i}$ is accomplished then the symbol currently scanned by the head is used to determine which subroutine $P_{j}$ is to be executed next. If the head is out of $w_{1}$ in such a moment, $A_{1}$ stops.

\section{3) $P$ is of the form $P_{1} ; P_{2}$}

where $P_{1}$ and $P_{2}$ are simple programs. Fictive variables can be introduced into $P_{1}$ and $P_{2}$ (e.g. using statements of the form $X:=X$ ) so that $P_{1}$ and $P_{2}$ contain the same identifiers as $P$. Then $2-D F T A_{P_{1}}$ and $A_{P_{2}}$ equivalent to $P_{1}$ and $P_{2}$, respectively, exist by the induction assumption. The serial composition of $\mathrm{A}_{\mathrm{P}_{1}}$ and $\mathrm{A}_{\mathrm{P}_{2}}$ is equivalent to $\mathrm{P}$ and this composition can be replaced by $\frac{1}{a}$ single $2-D F T$ by theorem 2 .

We have proved that for every program $P$ there is an equivalent 2-DFT, provided all variables were declared as input and output ones. For the general case when variables $x_{i_{1}}, \ldots, x_{i_{r}}$ are declared as the input ones and variables $x_{o_{1}}, \ldots, x_{o_{s}}$ as the output ones, it suffices to construct the serial composition of 2-DFTs $A_{\text {in }}, A_{P}, A_{\text {out }}$, where $A_{\text {in }}$ performs the input encoding $\left(X^{*}\right)^{r} \rightarrow\left(X^{*}\right)^{m}$ for $A_{P}$ by printing the input values into the appropriate tracks and leaving the remaining tracks empty, Aout on the other hand picks up the output values.

The serial composition of the automata performs the same mapping $\left(X^{*}\right)^{r} \rightarrow\left(X^{*}\right)^{5}$ as $P$ and can be replaced by a single 2-DFT.

References

1. J.E. Hopcroft and J.D. UlIman, An Approach to a Unified Theory of Automata, The Bell System Technical Journal 46 (1967), 1793-1829.

2. A.V. Aho, J.E. Hopcroft and J.D. Ullman, A General Theory of Translation, Mathematical Systems Theory 3 (1969), 193-221.

3. J.E. Hopcroft and J.D. Ullman, Formal Ianguages and Their Relation 
to Automata, Addison-Wesley 1969

4. A.V. Aho and J.D. Ullman, A Characterization of Two-Way Deterministic Classes of Languages, JCSS 4 (1970), 523-538.

5. V. Rajlich, Bounded-Crossing Transducers, Information and Control 27 (1975), 329-335.

6. D. Scott, Some Definitional Suggestions for Automata Theory, JCSS 1 (1967), 187-212.

7. G. Ausiello, Simple Programs on Strings and Their Decision Problems, University of Rome, R.75-17, 1975.

8. G. Ausiello and M. Moscarini, on the Complexity of Decision Problems for Classes of Simple Programs on Strings, Proceedings of GI -6 . Jahrestagung (1976), Informatik - Fachberichte, Springer Verlag, 1976. 